\title{
High-throughput application of Palindromic Sequence-Targeted (PST) PCR method for genomic fingerprinting and transposon display
}

\author{
Kalendar R.N. ${ }^{1}$, Bektayev R.T. ${ }^{2 *}$ \\ ${ }^{1}$ National Laboratory Astana, Nur-Sultan, Kazakhstan \\ ${ }^{2}$ National Center for biotechnology, Nur-Sultan, Kazakhstan \\ * email: bektayev@biocenter.kz
}

Genomic fingerprinting for various identification purposes has become a routine procedure to monitor biodiversity and control production, consumption and transportation of plants and plant-derived products. New molecular genetic methods for comparison of whole genomes or identification of selected genetic markers are continually being developed and compete with each other for their informative capacity, costs, and other properties. For this purpose, the original Palindromic SequenceTargeted (PST) PCR technique that can identify biological species in various population studies was developed. The high sensitivity and specificity of the method, provided by special thermocycling profile with the use of a combination of specially designed walking and sequence-specific primers were shown by the study results. The results allow us to propose PST-PCR as a method for gene-targeted genomic fingerprinting. It was demonstrated on the example of the Poaceae family using the VRN1 gene as a target. In this regard, multiple alignments of the $V R N 1$ gene exon 1 shows regions of varying conservation suitable for discrimination of all Poaceae species. The VRN1 gene has the potential to be a universal marker for fingerprinting of cereals and grasses. Alignment of DNA sequences of the VRN1 exons from various members of Poaceae revealed both conserved and variable regions. Primers targeting exon 1 were tested on all representatives from Phleum pratense available to the authors and universally led to amplification of the desired PCR product. DNA sequencing revealed the existence of sequence polymorphisms in the promoter and intron 1. Analysis of the molecular organization of the gene, including the lengths of the promoter and intron 1 and copynumber variation, allowed the description of polymorphisms suitable for gene-targeted genomic fingerprinting of all Poaceae species. Developed protocols may be used in the identification of lines and intraspecific and inter-species genetic variability in members of the Poaceae. 